\title{
Release of active peptidylarginine deiminase into the circulation during acute inflammation induced by coronary artery bypass surgery
}

This article was published in the following Dove Press journal: Journal of Inflammation Research

\begin{abstract}
Anne Vejlstrup'
Ann Merete Møller ${ }^{2}$ Claus Henrik Nielsen ${ }^{1,3, *}$ Dres Damgaard ${ }^{1,3, *}$

'Institute for Inflammation Research, Center for Rheumatology and Spine Diseases, Copenhagen University Hospital, Rigshospitalet, Copenhagen, Denmark; ${ }^{2}$ Department of Anaesthesiology, Herlev Hospital, Herlev, Denmark; ${ }^{3}$ Section for Periodontology, Microbiology and Community Dentistry, Department of Odontology, Faculty of Health and Medical Sciences, University of Copenhagen, Copenhagen, Denmark
\end{abstract}

*These authors contributed equally to this work
Correspondence: Dres Damgaard Institute for Inflammation Research, Center for Rheumatology and Spine Diseases, Copenhagen University Hospital, Rigshospitalet, Section 754I, Blegdamsvej 9, DK-2100 Copenhagen, Denmark

Tel +4535457l88

Email dres.damgaard@regionh.dk
Purpose: Peptidylarginine deiminase (PAD) catalyzes citrullination, a post-translational modification that can alter structure, function and antigenicity of proteins. Citrullination in the lungs due to smoking is believed to initiate an anti-citrulline immune response in rheumatoid arthritis. Citrullination in other inflamed organs has also been demonstrated, but it is not known whether smoking or inflammatory processes in general result in release of relevant amounts of PAD into the circulation with potential to cause citrullination of proteins at various anatomical sites. Coronary artery bypass grafting (CABG) using cardiopulmonary bypass (CPB) induces an acute systemic inflammation response. In the present study, we investigate whether smoking or acute systemic inflammation causes release of PAD into the circulation.

Patients and methods: This study included 36 patients with coronary heart disease (16 smokers and 20 non-smokers) undergoing CABG surgery with CPB. Circulating levels of PAD2 and PAD4, PAD activity, the neutrophil activation markers MPO, MMP-9 and lipocalin-2, the cytokines IL-6 and IL-10, and the chemokine CXCL8 were measured $2 \mathrm{hrs}$ preoperatively and $2 \mathrm{hrs}$ postoperatively.

Results: At baseline, serum PAD2 and PAD4 concentration did not differ between smokers and non-smokers. However, serum from non-smokers contained higher PAD activity than serum from smokers. Circulating PAD2 levels and PAD activity increased markedly in both groups after surgery, as did all neutrophil activation markers, cytokines and chemokine. PAD2 levels correlated with neutrophil activation markers, but not with cytokine and chemokine levels.

Conclusion: Blood levels of PAD2 did not differ significantly between smokers and nonsmokers, but smokers had decreased PAD activity in the circulation. PAD2 levels and PAD activity increased in blood during inflammation induced by CABG with CPB. This suggests that acute inflammation, ischemia or reperfusion, or a combination of these, leads to systemic spreading of enzymatically active PAD, which may affect protein function and induce generation of citrullinated self-antigens.

Keywords: citrullination, peptidylarginine deiminase, PAD, inflammation, coronary artery bypass grafting, CABG, cardiopulmonary bypass, $\mathrm{CPB}$

\section{Introduction}

Peptidylarginine deiminase (PAD) catalyzes the post-translational protein modification known as citrullination, which refers to the conversion of arginine residues into citrulline residues. Citrullination can alter the function of several proteins, including cytokines and chemokines, ${ }^{1,2}$ fibrinogen, ${ }^{3}$ nicotinamide-N-methyl transferase ${ }^{4}$ and transforming growth 
factor- $\beta,{ }^{5}$ and induce structural changes in proteins that generate autoantigenic neoepitopes, as observed in rheumatoid arthritis (RA) and type I diabetes. ${ }^{6,7}$ The human PAD enzyme family has five members (PADs $1-4$ and 6), of which PAD2 and PAD4 are expressed by cells of hematopoietic origin, eg, monocytes, lymphocytes and granulocytes. ${ }^{8}$ Neutrophils are considered a major source of PAD2 and PAD4, ${ }^{9-11}$ and PAD released from neutrophils is capable of citrullinating extracellular proteins. ${ }^{10,12}$ Prerequisites for PAD activation are a high calcium concentration, which is found in the extracellular space, ${ }^{13}$ and a redox potential that maintains the catalytic site in a reduced state. ${ }^{10,14}$ PAD and citrullinated proteins are abundant in the lungs of smokers and are suspected to be the initial source of citrullinated proteins that cause the anticitrulline immune response involved in RA. ${ }^{15-17}$ Chronic obstructive pulmonary disease ${ }^{18}$ and periodontitis ${ }^{19}$ are other examples of inflammatory conditions associated with increased local levels of citrullinated proteins. Indeed, protein citrullination appears to occur in inflammation in general. ${ }^{20}$ However, it is not known whether inflammation leads to release of PAD into the bloodstream, which may potentially cause citrullination of proteins in the circulation or at distant anatomical sites, thereby generating antigens that ignite autoimmune reactions.

Coronary artery bypass graft (CABG) surgery with cardiopulmonary bypass (CPB) is an example of a circumstance where acute systemic inflammation occurs, partly due to ischemia-reperfusion injury after aortic unclamping leading to activation of endothelial cells and neutrophils, and partly because neutrophils are activated after contact with artificial surfaces of the cannula and tubing connecting the patient to the CPB system. ${ }^{21-24}$

We investigate here whether smoking is associated with increased levels of circulating PAD2 and PAD4, or with increased PAD activity. Moreover, we examine whether acute inflammation such as that induced by CABG surgery can lead to the release of enzymatically active PAD into the systemic circulation.

\section{Material and methods}

\section{Patient selection}

The study included 36 patients with coronary heart disease who underwent conventional elective $\mathrm{CABG}$ at the Department of Thoracic Surgery, Copenhagen University Hospital Rigshospitalet. Patients below 18 years of age, pregnant women, and patients with cancer, autoimmune disorders or chronic inflammatory disorders were excluded.
Patient characteristics are shown in Table 1. The smokers included $(n=16)$ had all smoked a minimum of 10 cigarettes per day for at least 5 years and had smoked in the $48 \mathrm{hrs}$ before surgery. The non-smokers $(n=20)$ had all smoked $<100$ cigarettes ever. The study was approved by the Regional Ethics Committee H-3-2014-164 and was performed in compliance with the Declaration of Helsinki, and all patients gave written informed consent.

\section{Preoperative medication}

All patients had their aspirin and antiplatelet drugs paused before the surgery. All patients were receiving treatment with antihypertensives and antihypercholesterolemic agents before the surgery. Patients with type 2 diabetes were receiving treatment with either insulin or oral antidiabetics. One patient was receiving treatment with the anti-inflammatory prednisolone $(2.5 \mathrm{mg})$ before surgery.

\section{Anesthesia}

Anesthetic management was standardized. No premedication was given. Anesthesia was induced with fentanyl and propofol infusion. Propofol, sevofluran and remifentanil were titrated thereafter. Cisatracurium was administered before tracheal intubation. Some patients were given bolus $\mathrm{CaCl}$ and noradrenaline when coming off the CPB. Corticosteroids were not used in any of the patients during perioperative management. Cefuroxime $(1.5 \mathrm{~g})$ and gentamicin $(240 \mathrm{mg}$ ) were given perioperatively.

\section{Surgical procedure}

All CABG procedures were performed via a median sternotomy, and on-pump with $\mathrm{CPB}$ and extracorporeal circulation (ECC). Patients were fully heparinized to maintain an acquired coagulation time above $480 \mathrm{~s}$. This effect was reversed with protamine sulfate (1:1) after finalization of ECC. Cardiac arrest was achieved with cold blood cardioplegia, and ECC was performed under normothermia. Standard anesthetic techniques were used, and intraoperative monitoring was in accordance with guidelines. Standard guidelines for anti-coagulation therapy were followed.

\section{Serum sampling}

Blood samples were drawn $2 \mathrm{hrs}$ before surgery and $2 \mathrm{hrs}$ after surgery (from the closure of the skin) in $6 \mathrm{~mL}$ dry tubes (VACUETTE, Greiner Bio-One, Frickenhausen, Germany), from which serum was isolated. 
Table I Patient characteristics

\begin{tabular}{|c|c|c|c|}
\hline & & Non-smokers $\mathbf{n}=\mathbf{2 0}$ & Smokers $n=16$ \\
\hline \multicolumn{4}{|l|}{ Preoperative characteristics } \\
\hline Male/female & $\mathrm{n}$ & $19 / 1$ & $13 / 3$ \\
\hline Age, years & Mean (range) & $67.6(47-82)$ & $66.3(37-80)$ \\
\hline BMI, $\mathrm{kg} / \mathrm{m}^{2}$ & Mean $\pm S D$ & $28.4 \pm 3.2$ & $26.6 \pm 4.8$ \\
\hline \multicolumn{4}{|l|}{ Medical and surgical history } \\
\hline Hypertension & n (\%) & $15(75)$ & II (52.4) \\
\hline Hypercholesterolemia & n (\%) & $15(75)$ & II (52.4) \\
\hline Myocardial infarct & n (\%) & $7(35)$ & $5(23.8)$ \\
\hline Previous $\mathrm{PCl}$ & n (\%) & $5(25)$ & $3(14.3)$ \\
\hline T2DM & n (\%) & $8(40)$ & $5(23.8)$ \\
\hline COPD & n (\%) & I (5) & $3(14.3)$ \\
\hline \multicolumn{4}{|l|}{ LVEF } \\
\hline$<40 \%$ & n (\%) & $2(10)$ & I (6.25) \\
\hline $40-55 \%$ & $n(\%)$ & $8(40)$ & $11(68.8)$ \\
\hline$>55 \%$ & n (\%) & $10(50)$ & $4(25.0)$ \\
\hline $\mathrm{SpO}_{2}, \%$ & Mean $\pm S D$ & $98.2 \pm 1.3$ & $98.3 \pm 1.3$ \\
\hline \multicolumn{4}{|l|}{ Perioperative characteristics } \\
\hline CPB time, $\min$ & Mean \pm SD & $77.8 \pm 17.8$ & $818 \pm 25.8$ \\
\hline Aortic cross-clamp time, min & Mean \pm SD & $46 \pm 13.3$ & $46.9 \pm 15.3$ \\
\hline No. of grafts & Median (range) & $2(I-2)$ & $2(I-3)$ \\
\hline
\end{tabular}

Abbreviations: $\mathrm{BMI}$, body mass index; $\mathrm{PCl}$, percutaneous coronary intervention; LVEF, left ventricular ejection fraction; $\mathrm{SpO} \mathrm{O}_{2}$, peripheral capillary oxygen saturation; $\mathrm{CPB}$, cardiopulmonary bypass.

\section{Measurements of serum cytokines, markers of neutrophil activation and PAD}

Serum IL-6, IL-10 and CXCL-8 were measured using the Cytometric Bead Array Human Inflammatory Cytokine Kit II (Cat. no. 551811) from BD Biosciences (Franklin Lakes, NJ, USA) after dilution of $5 \mu \mathrm{L}$ serum in $20 \mu \mathrm{L}$ PBS according to the manufacturer's instructions. A FACS Canto II flow cytometer (BD Biosciences) was used for data acquisition, and the FCAPArray Software (SoftFlow, Burnsville, MN, USA) was used for analysis. Neutrophil activation markers were measured in serum diluted 1:50 in sample diluent following the manufacturer's instructions using the Luminex Bio-Plex ${ }^{\circledR} 200$ system (Bio-Rad Laboratories, Hercules, CA, USA) by means of the oneplex myeloperodidase (MPO) assay (R\&D, Abingdon, UK), and the two-plex MMP-9 and lipocalin-2/NGAL $(R \& D)$ assay. PAD2 was measured using an in-house PAD2specific assay on a Luminex platform. ${ }^{25}$ In brief, the murine monoclonal anti-PAD2 antibody DN2 was coupled to beads, and patient serum diluted 1:3 in sample buffer was added. After washing, biotinylated anti-PAD2 antibody $\mathrm{DN}^{25}$ and
PE-labelled streptavidin were used for detection. CV\% of spiked controls were $<15 \%$. PAD4 was measured in undiluted serum using a commercially available ELISA kit according to the manufacturer's instructions (SEF709Hu [N-terminal version], Cloud-Clone, TX, USA). Ability to detect PAD4 specifically was confirmed by spiking of rhPAD2 or rhPAD4 into serum, but low recovery $(<10 \%)$ was observed.

\section{Determination of PAD activity}

PAD activity in serum was determined using a previously described assay for citrullination of matrix-bound fibrinogen. ${ }^{13}$ In brief, Nunc ELISA plates (Roskilde, Denmark) were coated with fibrinogen $(1.0 \mu \mathrm{g} / \mathrm{mL})$. After three washes, wells were blocked with Tris-buffered saline containing $0.05 \%$ Tween 20 for 20 mins at room temperature. Serum was added (50 $\mu \mathrm{L} /$ well) and incubated for $4 \mathrm{hrs}$ at RT, with or without $1 \mathrm{mM}$ DTT and/or $25 \mathrm{mM}$ EDTA. After three washes in PBS containing $0.05 \%$ Tween 20, the wells were incubated for 90 mins with $100 \mu \mathrm{L}$ of murine anticitrullinated fibrinogen antibody (clone 20B2, Modiquest, 
Oss, Netherlands) $0.5 \mu \mathrm{g} / \mathrm{mL}$ in PBS containing $0.05 \%$ Tween. After three further washes, the wells were incubated with $100 \mu \mathrm{L}$ of HRP-conjugated polyclonal rabbit antimouse immunoglobulin antibodies (Dako, Glostrup, Denmark) diluted 1:1,000 in PBS, 0.05\% Tween. Finally, the plates were washed three times and developed with 3,3',5,5'-tetramethylbenzidine. The reaction was stopped after 15 mins with $\mathrm{H}_{2} \mathrm{SO}_{4}$ at a final concentration of $0.5 \mathrm{M}$.

\section{Statistical analyses}

The Mann-Whitney test was used to compare differences between groups using GraphPad Prism 7.02 (GraphPad Software, La Jolla, CA, USA). The Wilcoxon matchedpairs signed-rank test was used to determine differences before and after surgery. Spearman's correlation (Rs) coefficients and levels of significance were determined using GraphPad Prism. Two-tailed probability values $<0.05$ were considered significant.

\section{Results}

\section{Determination of circulating PAD2 and PAD4 levels}

We observed no significant difference in PAD2 or PAD4 levels between smokers and non-smokers before or after surgery (Figure 1A, B, D, and E). The circulating PAD2 levels of nearly all participants increased during surgery; the median level before surgery was $0.3 \mathrm{ng} / \mathrm{mL}$ (10-90 percentile range: $0.1-1 \mathrm{ng} / \mathrm{mL})$ compared to $1.15 \mathrm{ng} / \mathrm{mL}(0.3-5.12 \mathrm{ng} / \mathrm{mL})$ after surgery $(p<0.0001)$ (Figure $1 \mathrm{C})$. We found no significant
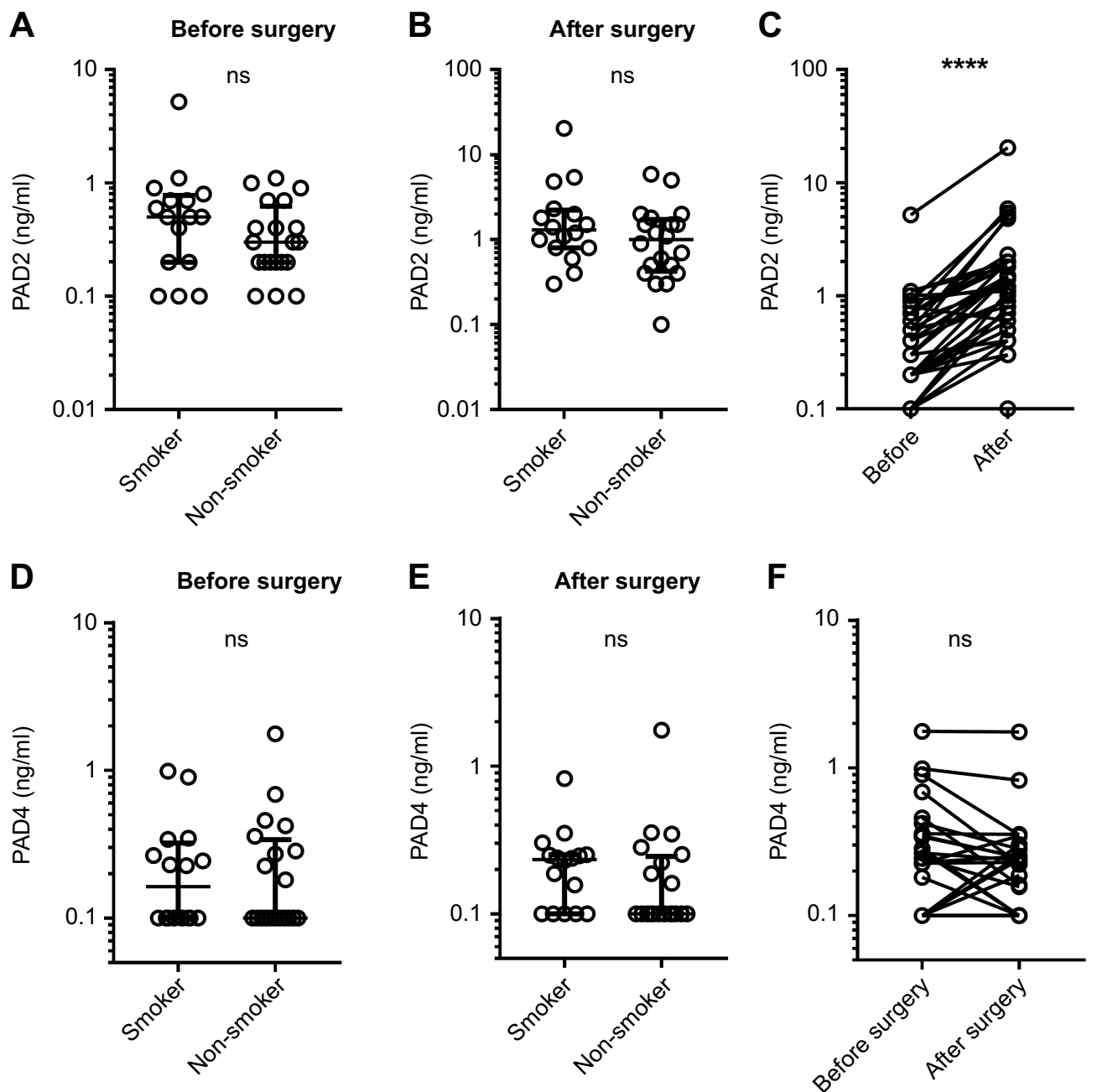

Figure I Circulating PAD2 and PAD4 levels before and after CABG surgery. Circulating levels of PAD2 and PAD4 were assessed in 16 smokers and 20 non-smokers before and after CABG surgery. (A) Serum PAD2 concentration before surgery. (B) Serum PAD2 concentration after surgery. (C) Serum PAD2 concentration of all patients before and after CABG. (D) Serum PAD4 concentration before surgery. (E) Serum PAD4 concentration after surgery. (F) Serum PAD4 concentration of all patients before and after CABG. Levels below the lower limit of detection of the two tests $(100 \mathrm{pg} / \mathrm{mL})$ were set to $100 \mathrm{pg} / \mathrm{mL}$. $* * * * p<0.000 \mathrm{I}$.

Abbreviations: ns, not significant; PAD, peptidylarginine deiminase; CABG, coronary artery bypass grafting. 
difference between PAD4 levels in the blood before and after surgery (Figure 1F).

\section{Catalytic activity of circulating PAD}

We next tested whether the circulating PAD was enzymatically active and observed higher PAD activity in serum from non-smokers than in serum from smokers before surgery (Figure 2A), which we did not expect. This difference was also present after surgery (Figure 2B). Since smoking has been reported to cause oxidation of GSH and cysteine in the blood, ${ }^{26}$ we examined
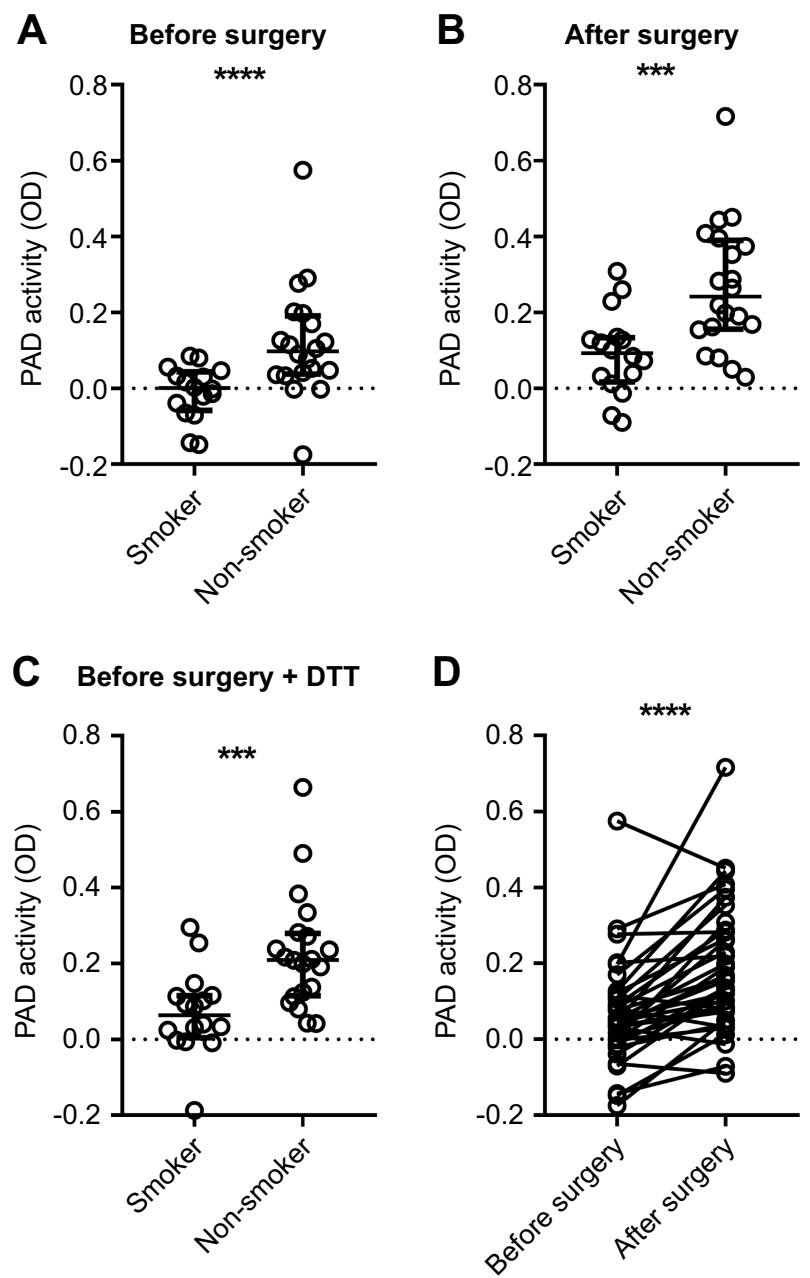

Figure 2 PAD activity in serum after CABG surgery. ELISA microtiter plates coated with human fibrinogen were incubated for 4 hrs with sera from 36 patients who had undergone CABG surgery, either with I mM DTT (+DTT) or without additives (no DTT). As negative control, each serum was tested in the presence of $25 \mathrm{mM}$ EDTA. (A) PAD activity in serum among smokers and non-smokers before surgery. (B) PAD activity in serum of smokers and non-smokers after surgery. (C) PAD activity at baseline in serum containing I mM DTT. (D) PAD activity of all untreated serum samples before and after surgery. Citrullination was measured using anti-citrullinated fibrinogen mAb (20B2) recognizing a citrullinated epitope in fibrinogen. Data are expressed as average OD of duplicate measurements after subtraction of the negative controls, and median and interquartile ranges of the dataset are shown. $* * * p<0.00 \mathrm{I}$, $* * * * *_{p}<0.000 \mathrm{I}$.

Abbreviations: PAD, peptidylarginine deiminase; CABG, coronary artery bypass grafting; DTT, dithiothreitol. whether PAD activity could be restored by addition of DTT, an exogenous reducing agent. PAD activity increased in both groups after addition of DTT, but the activity remained lower in smokers than in non-smokers (Figure 2C). As illustrated in Figure 2D, CABG surgery caused a marked increase in catalytic activity of circulating PAD in nearly all patients tested, both for smokers and non-smokers $(p<0.001)$.

\section{Effect of CABG surgery on inflammatory mediators and neutrophil degranulation}

To determine whether the increase in PAD2 levels and PAD activity observed during CABG surgery reflected the state of inflammation, we quantified circulating levels of the proinflammatory cytokine IL-6, the anti-inflammatory cytokine IL-10, and the chemokine CXCL-8 (Figure 3A-C). The levels of all three molecules increased after surgery (Figure $3 \mathrm{~A}-\mathrm{C}$ ). To address the degree of neutrophil activation, we quantified MPO, MMP-9 and lipocalin-2, as markers of degranulation of the primary, secondary and tertiary granules, respectively. The levels of all three markers were elevated after surgery (Figure 3D-F).

Before surgery, the levels of PAD2 did not correlate with any of the biomarkers, whereas a significant correlation was found between circulating PAD2 and MPO levels after surgery ( $p=0.03$; Table 2 ). After surgery, we observed a trend toward correlation between PAD2 and the two other markers of neutrophil activation, whereas no association was observed with IL-6, IL-10 or CXCL8. When the basal levels of all six biomarkers before surgery were subtracted from those observed after surgery (shown as " $\Delta$ Surgery" in Table 2 ), the change in circulating PAD2 levels correlated with the changes in both MPO and lipocalin-2 levels, but not with the change in IL-6, IL-10 or CXCL-8. No associations were observed between PAD4 and the six biomarkers before or after surgery (data not shown).

\section{Discussion}

Citrullination alters the function and antigenicity of many proteins ${ }^{2,4,27}$ and is thought to play a critical role in the pathogenesis of certain autoimmune diseases, especially RA. ${ }^{7}$ Citrullination in this respect is thought to occur at the very sites of PAD release, eg, in the lungs, periodontal tissue or gut. ${ }^{15,19,28}$ In this study, we put forward the hypothesis that PAD is released into the circulation following smoking or acute inflammation. 

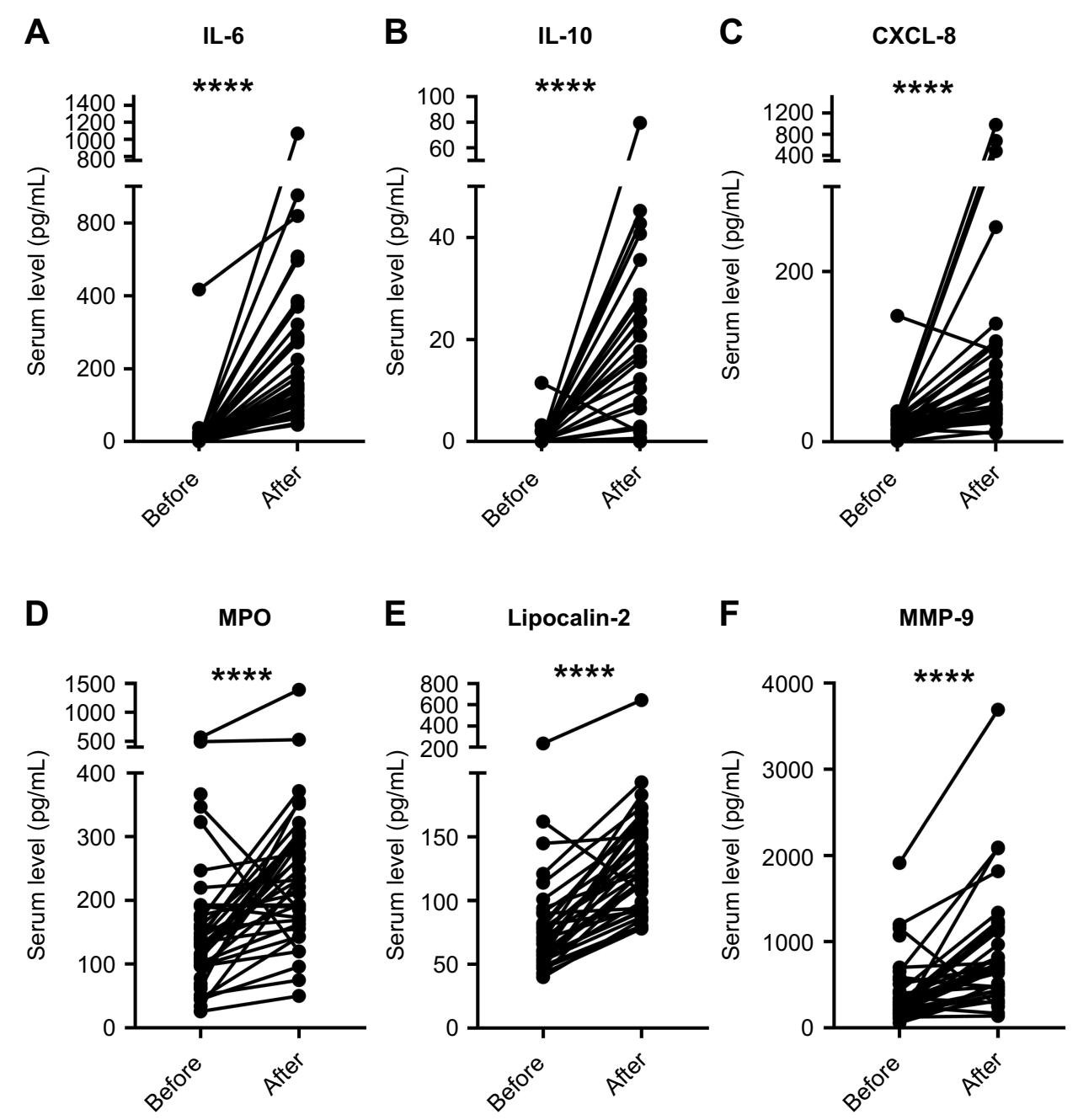

Figure 3 Circulating levels of cytokines and markers of neutrophil degranulation before and after CABG. Serum levels of (A) IL-6, (B) IL-I0, (C) CXCL-8, (D) MPO, (E) lipocalin-2, and (F) MMP-9 before and after surgery. $* * * * p<0.0001$.

Abbreviations: CABG, coronary artery bypass grafting; MPO, myeloperoxidase; MMP, matrix metallopeptidase.

Table 2 Association between PAD2 levels and inflammatory markers in serum

\begin{tabular}{|l|l|l|l|l|l|l|}
\hline & IL-6 & IL-10 & CXCL-8 & MPO & MMP-9 & Lipocalin-2 \\
\hline Before & $r_{s}=-0.12 p=0.49$ & $r_{s}=-0.04 p=0.84$ & $r_{s}=-0.08 p=0.64$ & $r_{s}=0.024 p=0.88$ & $r_{s}=-0.01 p=0.97$ & $r_{s}=0.014 p=0.93$ \\
After & $r_{s}=0.09 p=0.56$ & $r_{s}=-0.014 p=0.93$ & $r_{s}=0.19 p=0.25$ & $r_{s}=0.36, p=0.03$ & $r_{s}=0.32 p=0.056$ & $r_{s}=0.33 p=0.052$ \\
$\Delta$ Surgery* & $r_{s}=0.15 p=0.39$ & $r_{s}=0.10 p=0.54$ & $r_{s}=0.12 p=0.50$ & $r_{s}=0.35 p=0.039$ & $r_{s}=0.32 p=0.055$ & $r_{s}=0.51 p=0.001$ \\
\hline
\end{tabular}

Note: ${ }^{*} \Delta$ Surgery describes the increase from pre- to post-surgery blood levels. Statistically significant $p$-values are marked in bold.

Abbreviations: CABG, coronary artery bypass grafting; MPO, myeloperoxidase; MMP, matrix metallopeptidase.

PAD2 was present in detectable concentrations in the blood from most patients before surgery, and was found to be markedly increased after surgery. This was not the case for PAD4 levels. We have previously found detectable blood levels of PAD2 in less than a third of normal blood donors, ${ }^{25}$ but to our knowledge, no other studies have examined circulating PAD2 in relation to disease or inflammation. Although smokers tended to have slightly higher blood levels of PAD2 than non-smokers, there was a great overlap between our groups. Increased amounts of PAD2 along with increased numbers of macrophages have previously been shown in the lungs of smokers. ${ }^{16,17}$ Our data indicate that this PAD2 enters the bloodstream to only a limited extent. This may be because it does not cross the alveolar epithelium or is rapidly degraded locally. It is also possible that alveolar macrophages had not been 
sufficiently stimulated for PAD release before blood sampling in this study.

Importantly, the PAD contained in the serum samples was enzymatically active in many cases. Thus, PAD released into the circulation during local inflammation may citrullinate extracellular proteins, which potentially modify their functional properties or render proteins antigenic. Surprisingly, we found that the PAD contained in the blood of smokers was less active than that of nonsmokers. The PAD activity of serum from smokers could be restored by addition of the reducing agent DTT, which suggests that PAD is inactivated by oxidation in the blood of smokers, presumably by reactive oxygen species released as a consequence of smoking. ${ }^{26}$ It is possible that circulating inactive PAD can be reactivated at anatomical sites with a reducing environment. Thus, PAD activity can be restored in synovial fluid from RA patients after addition of a reducing agent, ${ }^{10}$ but to our knowledge, this is the first time that catalytic activity has been observed in serum without addition of reducing agents.

The fact that only PAD2 levels increased during surgery in this study suggests that PAD2 is predominantly responsible for the observed PAD activity in the blood. Neutrophils are a predominant source of PAD2 and PAD4, 9,10,12 and our finding that the increase in PAD2 levels during surgery correlated with the increase in neutrophil activation markers but not with changes in proinflammatory cytokines - is in keeping with this. This agrees with previous findings that extracellular activity of PAD released from neutrophils in vitro is primarily mediated by PAD2. ${ }^{29}$ PAD2 is rapidly released from neutrophils on stimulation, ${ }^{12}$ and with blood sampling 2 hrs after surgery, it is likely that the PAD2 released during the surgical procedure was pre-formed rather than synthesized de novo. The measurements of PAD4 in this study should be interpreted with caution, however, since low recovery was observed in the PAD4 ELISA after spiking of PAD4 into serum.

CABG surgery is based on the use of the CPB, which is known to induce ischemia, neutrophil activation, and a systemic inflammatory response which exceeds that observed after other major surgical procedures. ${ }^{21,23}$ We cannot rule out that the neutrophil activation observed in this study and any consequent PAD release were caused by sheer mechanical stress or hypoxia. However, extracellular PAD activity is observed as a consequence of inflammatory stimuli, ${ }^{30}$ so we propose that PAD2 release and an increase in circulating PAD activity are features of acute inflammation in a broader sense. This remains to be tested in other inflammatory conditions.

\section{Conclusion}

PAD2 is released into circulation during CABG surgery with $\mathrm{CPB}$, and the PAD released is enzymatically active. The increase in PAD2 levels correlated with an increase in neutrophil activation markers, indicating that neutrophils were the predominant source of PAD2. Smoking had no statistically significant influence on circulating PAD2 levels, and the catalytic activity of circulating PAD was unexpectedly decreased among smokers. The implications of this remain to be investigated.

\section{Acknowledgments}

The study was supported by the Research Foundation at Rigshospitalet, the Brødrene Hartmanns Foundation, and the Danish Council for Independent Research. The authors wish to thank Alistair Reeves for editing the manuscript. $\mathrm{DD}$ and $\mathrm{CHN}$ share the senior authorship.

\section{Disclosure}

The authors report no conflicts of interest with this work.

\section{References}

1. Proost P, Loos T, Mortier A, et al. Citrullination of CXCL8 by peptidylarginine deiminase alters receptor usage, prevents proteolysis, and dampens tissue inflammation. $J$ Exp Med. 2008;205:2085-2097. doi:10.1084/jem.20080305

2. Moelants EA, Mortier A, Grauwen K, Ronsse I, Van DJ, Proost P. Citrullination of TNF-alpha by peptidylarginine deiminases reduces its capacity to stimulate the production of inflammatory chemokines. Cytokine. 2013;61:161-167. doi:10.1016/j.cyto.2012.09.011

3. Nakayama-Hamada M, Suzuki A, Furukawa H, Yamada R, Yamamoto K. Citrullinated fibrinogen inhibits thrombin-catalysed fibrin polymerization. J Biochem. 2008;144:393-398. doi:10.1093/ $\mathrm{jb} / \mathrm{mvn} 079$

4. Nemmara VV, Tilvawala R, Salinger AJ, et al. Citrullination inactivates nicotinamide-N-methyltransferase. ACS Chem Biol. 2018;13:2663-2672. doi:10.1021/acschembio.8b00578

5. Sipila KH, Ranga V, Rappu P, et al. Extracellular citrullination inhibits the function of matrix associated TGF-beta. Matrix Biol. 2016. doi:10.1016/j.matbio.2016.02.008

6. Rondas D, Crevecoeur I, D'Hertog W, et al. Citrullinated glucose-regulated protein 78 is an autoantigen in type 1 diabetes. Diabetes. 2015;64:573-586. doi:10.2337/db14-0621

7. Schellekens GA, de Jong BA, van den Hoogen FH, van de Putte LB, van Venrooij WJ. Citrulline is an essential constituent of antigenic determinants recognized by rheumatoid arthritis-specific autoantibodies. J Clin Invest. 1998;101:273-281. doi:10.1172/JCI1316

8. Vossenaar ER, Zendman AJ, van Venrooij WJ, Pruijn GJ. PAD, a growing family of citrullinating enzymes: genes, features and involvement in disease. Bioessays. 2003;25:1106-1118. doi:10.1002/bies.10357

9. Asaga H, Nakashima K, Senshu T, Ishigami A, Yamada M Immunocytochemical localization of peptidylarginine deiminase in human eosinophils and neutrophils. J Leukoc Biol. 2001;70:46-51.

10. Damgaard D, Bjorn ME, Steffensen MA, Pruijn GJ, Nielsen CH. Reduced glutathione as a physiological co-activator in the activation of peptidylarginine deiminase. Arthritis Res Ther. 2016;18:102. doi:10.1186/s13075-016-1000-7 
11. Zhou Y, Di PT, Sims GP, Mittereder N, Mustelin T. Characterization of the hypercitrullination reaction in human neutrophils and other leukocytes. Mediators Inflamm. 2015;2015:236451. doi:10.1155/2015/125380

12. Spengler J, Lugonja B, Jimmy YA, et al. Release of active peptidyl arginine deiminases by neutrophils can explain production of extracellular citrullinated autoantigens in rheumatoid arthritis synovial fluid. Arthritis Rheumatol. 2015;67:3135-3145. doi:10.1002/art.39313

13. Damgaard D, Senolt L, Nielsen M, Pruijn G, Nielsen CH. Demonstration of extracellular peptidylarginine deiminase (PAD) activity in synovial fluid of patients with rheumatoid arthritis using a novel assay for citrullination of fibrinogen. Arthritis Res Ther. 2014;16:498. doi:10.1186/s13075-014-0498-9

14. Damgaard D, Bjorn ME, Jensen PO, Nielsen CH. Reactive oxygen species inhibit catalytic activity of peptidylarginine deiminase. J Enzyme Inhib Med Chem. 2017;32:1203-1208. doi:10.1080/ 14756366.2017.1368505

15. Klareskog L, Padyukov L, Alfredsson L. Smoking as a trigger for inflammatory rheumatic diseases. Curr Opin Rheumatol. 2007;19:49-54. doi:10.1097/BOR.0b013e32801127c8

16. Makrygiannakis D, Hermansson M, Ulfgren AK, et al. Smoking increases peptidylarginine deiminase 2 enzyme expression in human lungs and increases citrullination in BAL cells. Ann Rheum Dis. 2008;67:1488-1492. doi:10.1136/ard.2007.075192

17. Damgaard D, Friberg Bruun NM, Quisgaard GM, Palarasah Y, Svane-Knudsen V, Nielsen CH. Smoking is associated with increased levels of extracellular peptidylarginine deiminase 2 (PAD2) in the lungs. Clin Exp Rheumatol. 2015;33:405-408.

18. Lugli EB, Correia RE, Fischer R, et al. Expression of citrulline and homocitrulline residues in the lungs of non-smokers and smokers: implications for autoimmunity in rheumatoid arthritis. Arthritis Res Ther. 2015;17:9. doi:10.1186/s13075-015-0520-x

19. Engstrom M, Eriksson K, Lee L, et al. Increased citrullination and expression of peptidylarginine deiminases independently of $\mathrm{P}$. gingivalis and A. actinomycetemcomitans in gingival tissue of patients with periodontitis. J Transl Med. 2018;16:214. doi:10.1186/ s12967-018-1588-2

20. Makrygiannakis D, af Klin E, Lundberg IE, et al. Citrullination is an inflammation-dependent process. Ann Rheum Dis. 2006;65:12191222. doi:10.1136/ard.2005.049403
21. Jongman RM, Zijlstra JG, Kok WF, et al. Off-pump CABG surgery reduces systemic inflammation compared with on-pump surgery but does not change systemic endothelial responses: a prospective randomized study. Shock. 2014;42:121-128. doi:10.1097/SHK.000000 0000000190

22. Andresen TK, Svennevig JL, Videm V. Soluble VCAM-1 is a very early marker of endothelial cell activation in cardiopulmonary bypass. Perfusion. 2002;17:15-21. doi:10.1191/0267659102pf531oa

23. Toft P, Nielsen CH, Tonnesen E, Hansen TG, Hokland M. Changes in adhesion molecule expression and oxidative burst activity of granulocytes and monocytes during open-heart surgery with cardiopulmonary bypass compared with abdominal surgery. Eur $J$ Anaesthesiol. 1998; 15:345-353.

24. Denizot Y, Leguyader A, Cornu E, et al. Release of soluble vascular endothelial growth factor receptor-1 (sFlt-1) during coronary artery bypass surgery. J Cardiothorac Surg. 2007;2:38. doi:10.1186/17498090-2-38

25. Damgaard D, Palarasah Y, Skjodt K, et al. Generation of monoclonal antibodies against peptidylarginine deiminase 2 (PAD2) and development of a PAD2-specific enzyme-linked immunosorbent assay. J Immunol Methods. 2014;405:15-22. doi:10.1016/j. jim.2013.12.008

26. Moriarty SE, Shah JH, Lynn M, et al. Oxidation of glutathione and cysteine in human plasma associated with smoking. Free Radic Biol Med. 2003;35:1582-1588.

27. Mortier A, Van DJ, Proost P. Regulation of chemokine activity by posttranslational modification. Pharmacol Ther. 2008;120:197-217. doi:10.1016/j.pharmthera.2008.08.006

28. Bennike TB, Ellingsen T, Glerup H, et al. Proteome analysis of rheumatoid arthritis gut mucosa. J Proteome Res. 2017;16:346-354. doi:10.1021/acs.jproteome.6b00598

29. Zhou Y, Chen B, Mittereder N, et al. Spontaneous secretion of the citrullination enzyme PAD2 and cell surface exposure of PAD4 by neutrophils. Front Immunol. 2017;8:1200. doi:10.3389/fimmu.2017.01200

30. Blachere NE, Parveen S, Fak J, Frank MO, Orange DE. Inflammatory but not apoptotic death of granulocytes citrullinates fibrinogen. Arthritis Res Ther. 2015;17:369. doi:10.1186/s13075015-0890-0
Journal of Inflammation Research

\section{Publish your work in this journal}

The Journal of Inflammation Research is an international, peerreviewed open-access journal that welcomes laboratory and clinical findings on the molecular basis, cell biology and pharmacology of inflammation including original research, reviews, symposium reports, hypothesis formation and commentaries on: acute/chronic inflammation; mediators of inflammation; cellular processes; molecular mechanisms; pharmacology and novel anti-inflammatory drugs; clinical conditions involving inflammation. The manuscript management system is completely online and includes a very quick and fair peerreview system. Visit http://www.dovepress.com/testimonials.php to read real quotes from published authors. 